Rev. Adm. Saúde Vol. 17, № 69, Out. - Dez. 2017

http://dx.doi.org/10.23973/ras.69.64

ARTIGO ORIGINAL

\title{
Ferramentas gerenciais na prática de enfermeiros da atenção básica em saúde
}

Management tools in the practice of nurses of basic health care

\author{
Samuel Andrade de Oliveira ${ }^{1}$, Maria de Lourdes de Almeida ${ }^{2}$, Marieta \\ Fernandes Santos ${ }^{3}$, Adriana Zilly ${ }^{4}$, Aida Maris Peres ${ }^{5}$, Fernanda Ludmilla \\ Rossi Rocha ${ }^{6}$
}

1. Mestrando de enfermagem da Escola de Enfermagem de Ribeirão Preto, USP

2. Doutora em enfermagem. Docente da Universidade Estadual do Oeste do Paraná, Foz do Iguaçu, PR

3. Doutora em enfermagem. Docente da Universidade Estadual do Oeste do Paraná, Foz do Iguaçu PR

4. Doutora em ciências biológicas. Docente da Universidade Estadual do Oeste do Paraná, Foz do Iguaçu PR

5. Doutora em enfermagem. Docente do Departamento de Enfermagem da Universidade Federal do Paraná, Curitiba PR

6. Doutora em enfermagem. Escola de Enfermagem de Ribeirão Preto, USP

\section{RESUMO}

Introdução. Para que a prestação de serviços à saúde seja de qualidade, se faz necessário que a assistência e a gerência estejam interligadas na execução do trabalho de enfermagem. Sendo assim, o objetivo foi identificar e caracterizar as ferramentas gerenciais utilizadas pelos enfermeiros que atuam na atenção básica à saúde para o gerenciamento das ações de enfermagem e saúde. Material e métodos. Pesquisa de abordagem qualitativa do tipo descritiva exploratória. Participaram da pesquisa 40 enfermeiros que atuam na atenção primária à saúde de um município do sul do Brasil, região de tríplice fronteira. Os dados foram coletados mediante entrevistas semiestruturadas e 
analisados conforme a técnica do discurso do sujeito coletivo. Resultados. Os enfermeiros da atenção primária à saúde entendem ferramentas gerenciais como meio de organização e direcionamento dos serviços de saúde, sendo referido seu uso diário na prática profissional. Porém, uma parte dos enfermeiros relatou ainda não utilizar ferramentas gerenciais ou realizam o uso de forma empírica, ou seja, ainda não há uma reflexão na prática diária sobre o uso dessas ferramentas. Entre as ferramentas utilizadas por estes enfermeiros foram citadas as de organização, controle, avaliação e direcionamento dos serviços. Conclusão. Apesar de os enfermeiros entenderem a relevância de desenvolver competências gerenciais e conhecer ferramentas de gestão para auxiliá-los no processo gerencial, torna-se necessário que estes profissionais se desvinculem das ações embasadas apenas no conhecimento empírico e tornem a sua prática crítica e reflexiva.

Palavras-chave: gestão em saúde, administração de serviços de saúde, atenção primária à saúde, serviços de enfermagem, enfermagem.

\begin{abstract}
Introduction. In order to provide quality health services, it is necessary that care and management be interconnected in the execution of nursing work. Thus, the objective was to identify and characterize the managerial tools used by nurses who work in basic health care for the management of nursing and health actions. Materials and methods. Qualitative exploratory descriptive research. A total of 40 nurses working in primary health care in a city in the south of Brazil, a region with a triple border, participated in the study. The data were collected through semi-structured interviews and analyzed according to the technique of the Collective Subject Discourse. Results and Discussion. Nurses of Primary Health Care understand management tools as a means of organizing and directing health services, and their daily use in professional practice is mentioned. However, some nurses reported that they do not use management tools or use them in an empirical way, that is, there is still no reflection in daily practice about the use of these tools. Among the tools used by these nurses were those of organization, control, evaluation and direction of services. Conclusion. Although nurses understand the importance of developing managerial skills and know management tools to assist them in the managerial process, it is necessary that these professionals dissociate themselves from actions based only on empirical knowledge and make their practice critical and reflexive.
\end{abstract}

Keywords: health management, health services administration, primary health care, nursing services, nursing. 


\section{INTRODUÇÃO}

A enfermagem se destaca cada vez mais na área da saúde, seja no contexto nacional ou internacional. Isto se deve ao fato de que o enfermeiro tem assumido cada vez mais um papel decisivo na resolução dos problemas de saúde da população. O sistema de saúde local tem o cuidado de enfermagem como fator primordial, com reflexo para os níveis regional e nacional, pois o enfermeiro tem assumido posições de liderança nos cenários de saúde que podem ser definidores da qualidade do cuidado prestado ${ }^{1}$.

Atualmente, tem-se o entendimento de que o trabalho do enfermeiro compreende várias dimensões, sendo estas o cuidado/assistência, ensino/educação, pesquisa e gestão/administração². Outro processo de trabalho do enfermeiro se refere à participação política, dimensão presente no cotidiano destes profissionais e que permeia os demais processos de trabalho, porém ainda pouco percebida pelos indivíduos e pouco explorada na pesquisa em enfermagem ${ }^{3}$.

Considera-se que, para que a prestação de serviços à saúde seja de qualidade, a assistência e a gerência estejam interligadas na execução do trabalho de enfermagem, pois, a divergência entre a dimensão assistencial e gerencial compromete a qualidade do cuidado e gera conflitos no trabalho do enfermeiro ${ }^{4-6}$.

Como foi dito, por ser responsável pela assistência que é prestada ao cliente, os enfermeiros desempenham funções que são assistenciais e administrativas para promover o cuidado. Estes profissionais necessitam adaptar-se continuamente a fim de articular essas duas funções no seu processo de trabalho ${ }^{6,7}$.

Vale destacar que o processo de trabalho gerencial do enfermeiro está condicionado ao contexto social e econômico do país, ao modelo de atenção à saúde adotado na instituição, às necessidades de saúde da população local, ao dimensionamento de pessoal de enfermagem, à política de saúde e à forma como este profissional insere-se no sistema de saúde vigente ${ }^{8}$.

Frente a isto, torna-se importante ressaltar que, com as transformações no mundo do trabalho ocorridas nas últimas décadas exige-se um gerenciamento voltado à compreensão e consideração da realidade política, econômica e social, o que é fundamental no processo de consolidação do Sistema Único de Saúde (SUS) e consequentemente nas estratégias a serem utilizadas para sua gestão ${ }^{9}$.

Na prática do gerenciamento, o enfermeiro, como coordenador e articulador do processo de cuidar, utiliza as bases ideológicas e teóricas de administração clássica e a prática de gerenciamento de recursos. Para tanto, as ferramentas gerenciais são de fundamental importância, tendo em vista que contribuem para a organização e avaliação dos serviços de saúde/enfermagem e, em consequência, para a melhoria da qualidade da atenção à saúde ofertada aos cidadãos ${ }^{10,11}$. 
O enfermeiro ainda conta com uma série de ferramentas e instrumentos que podem embasar sua prática, como as teorias administrativas, o processo de trabalho, a ética no gerenciamento, os saberes sobre cultura e poder organizacional, qualidade de vida no trabalho, saúde do trabalhador, leis trabalhistas, gerenciamento de pessoas, dimensionamento de pessoal, gerenciamento de recursos materiais, custos, recursos físicos, sistema de informação e processo decisório, a capacidade de negociação e a disposição para o trabalho em equipe $8,10,11$.

Alguns autores têm usado o termo instrumento ${ }^{7,11}$ ou ferramentas gerenciais ${ }^{10,12}$ para referirem ao arsenal de apoio à função gerencial do enfermeiro. Segundo o dicionário Houaiss da língua portuguesa ${ }^{13}$, os dois termos são sinônimos e referem-se a instrumento como algo que é utilizado para conseguir um resultado e a todo meio de conseguir um fim. Também diz respeito a utensílio, máquina, aparelho que serve para executar uma obra ou fazer uma operação. Sobre o termo ferramenta, o conceito é semelhante: qualquer instrumento utilizado para a realização de um trabalho, ou utensílio empregado nas artes ou ofícios, o conjunto desses utensílios ${ }^{13}$. Nesta pesquisa, optou-se em utilizar o termo ferramentas gerenciais para descrever os artefatos que os enfermeiros podem utilizar para apoiar o seu processo de trabalho gerencial.

Apoiado neste contexto e considerando que o conhecimento sobre as ferramentas gerenciais utilizadas pelo enfermeiro pode tornar sua prática gerencial mais acertada e sistematizada, principalmente no que diz respeito à sua prática na atenção básica à saúde, esta pesquisa pode favorecer reflexões, discussões e avanços no processo de trabalho gerencial do enfermeiro.

Frente a este desafio, surgem como questões de pesquisa as indagações: quais são as ferramentas gerenciais utilizadas pelos enfermeiros que atuam na atenção básica à saúde? Qual é a percepção destes profissionais acerca destas ferramentas? Desta forma, esta pesquisa teve como objetivo identificar e caracterizar as ferramentas gerenciais utilizadas pelos enfermeiros que atuam na atenção básica à saúde.

\section{MATERIAL E MÉTODOS}

O presente estudo constitui-se em uma pesquisa de abordagem qualitativa, do tipo descritiva exploratória, realizada em um município do interior do estado do Paraná. A população foi composta por 52 enfermeiros assistenciais que trabalhavam nas unidades de Atenção Primária à Saúde do município e o cálculo amostral evidenciou uma amostra de 46 enfermeiros. Para tal, foi utilizada proporção máxima, com nível de confiança de $95 \%$ e erro de $5 \%$.

A pesquisa atingiu $86,96 \%$ da amostra indicada, já que 40 enfermeiros participaram do estudo. Todos os enfermeiros foram convidados a participar da pesquisa, sendo que não foram incluídos apenas os enfermeiros que estavam de licença, férias, ou aqueles que não aceitaram participar da pesquisa. Foi utilizado como critério de inclusão: possuir experiência mínima de seis meses 
de atuação em Unidades Básicas de Saúde, o que se justifica pela necessidade de estes profissionais conhecerem a dinâmica do processo de trabalho da enfermagem nestes serviços.

Inicialmente, no intuito de validar o instrumento de coleta de dados a ser utilizado (roteiro de entrevistas elaborado pelos autores), foi realizado um préteste em uma das unidades de saúde com três enfermeiros para verificar se o instrumento respondia ao que se esperava no estudo. Após o pré-teste, realizou-se a coleta de dados, ocorrida no primeiro semestre de 2013 por meio de entrevistas semiestruturadas, que foram gravadas com a autorização dos participantes. Antes de iniciar as entrevistas, os participantes eram orientados sobre os objetivos do estudo e indagados sobre seu interesse em participar; caso concordassem, assinavam o Termo de Consentimento Livre e Esclarecido, o qual também solicitava autorização dos trabalhadores para a gravação das suas falas.

As entrevistas foram realizadas nos consultórios de enfermagem das unidades de saúde e tiveram duração de 30 minutos aproximadamente, com utilização de um gravador digital. Posteriormente, as falas dos participantes foram transcritas e organizadas com o uso do software Qualiquantisoft, utilizado como repositório de dados.

Para a análise dos dados, seguiu-se a técnica do discurso do sujeito coletivo $\mathrm{DSC}^{14}$, sendo as entrevistas norteadas pelas questões e pelos temas descritos no Quadro 1. O software Qualiquantisoft foi desenvolvido pelos mesmos autores da técnica do $\mathrm{DSC}^{14}$, em parceria com a Universidade de São Paulo e com uma empresa de informática, e tem como objetivo facilitar o desenvolvimento de pesquisas em que se utiliza a técnica ${ }^{15}$.

Quadro 1. Descritivo dos temas e questões norteadoras. Município do sul do Brasil, 2013.

\begin{tabular}{|l|l|}
\hline Temas & Questões norteadoras \\
\hline $\begin{array}{l}\text { Conceito de ferramentas } \\
\text { gerenciais }\end{array}$ & O que é uma ferramenta gerencial? \\
\hline $\begin{array}{l}\text { Utilização das ferramentas } \\
\text { gerenciais na prática }\end{array}$ & $\begin{array}{l}\text { Você utiliza ferramentas gerenciais no seu } \\
\text { cotidiano de trabalho? }\end{array}$ \\
\cline { 2 - 2 } & $\begin{array}{l}\text { Quais são as ferramentas gerenciais que você } \\
\text { utiliza na sua prática profissional? }\end{array}$ \\
\hline
\end{tabular}

A técnica do DSC organiza e tabula os dados de uma pesquisa qualitativa, pela análise dos depoimentos individuais que expressam o pensamento de uma coletividade. Após a análise do material (discurso individual), retiram-se de 
cada discurso individual as Ideias Centrais (IC) e/ou Ancoragens (AC) e suas correspondentes Expressões Chaves $(E C H)$ parecidas, obtendo-se um ou diversos discursos, os quais representam os discursos coletivos ${ }^{14}$.

As expressões chaves são partes, trechos ou transcrições literais de cada depoimento. É a partir do conteúdo das ECH que é construído o DSC. As IC são expressões linguísticas que revelam e descrevem, da forma mais resumida, precisa e fiel possível, o sentido de cada um dos discursos analisados e de cada conjunto de $\mathrm{ECH}$ semelhantes. A AC é uma figura metodológica, que é a manifestação linguística clara de uma teoria, ou ideologia, ou crença do sujeito que discursa ${ }^{14}$.

Nesta metodologia, as AC somente serão utilizadas quando forem encontradas no material verbal marcas discursivas explícitas dessas ideologias ${ }^{14}$. Esta figura de linguagem não foi utilizada, pois não foi encontrada nos resultados da presente pesquisa. Para os autores, as IC podem ser descrições diretas revelando o que foi dito, ou descrições indiretas sobre o tema do discurso do sujeito. Em síntese, o conjunto dos discursos individuais forma o pensamento de uma coletividade ${ }^{14}$.

Em relação aos aspectos éticos, o estudo ora apresentado foi aprovado pelo Comitê de Ética em Pesquisa de uma universidade pública sob registro $\mathrm{n}^{\circ}$ CAAE 09146312.0.0000.0107 e Parecer nํ2 235/2012.

\section{RESULTADOS}

Da população de 52 enfermeiros que trabalhavam nas UBS na ocasião do estudo participaram da pesquisa $40(76,9 \%)$ enfermeiros. Oito enfermeiros se encontravam de férias durante a realização da pesquisa, três não aceitaram participar e um tinha menos de seis meses de experiência.

O perfil sociodemográfico dos entrevistados apontou que $50 \%$ (20) tinham idades entre 30 a 39 anos e $90 \%$ (36) pertenciam ao sexo feminino. Quanto ao tempo de formação $62,5 \%$ (25) dos entrevistados tinham de um a oito anos de formação e 27\% (11) possuíam de um a seis anos de experiência na Atenção Básica. Em relação à área de atuação, o maior número se encontrou nas USF dentro das ESF, isto é $67,5 \%$ (27). Sobre a realização de pós-gradução, $90 \%$ (36) dos participantes já haviam cursado, sendo a maioria em Saúde da Família, seguida pelas áreas de saúde pública e/ou Atenção Básica.

Apresentam-se a seguir, os resultados da construção dos discursos do sujeito coletivo (DSC), e suas IC correspondentes. As entrevistas foram utilizadas como matéria-prima para compor os discursos formulados. Os DSC foram construídos com as IC selecionadas dentre todas que surgiram na pesquisa. Essa seleção ocorreu de acordo com a frequência das respostas dos enfermeiros dentro de cada IC formulada.

Para a identificação das ferramentas gerenciais utilizadas na prática do enfermeiro que atua na atenção básica à saúde, primeiramente perguntou-se 
aos participantes a sua concepção sobre as ferramentas gerenciais para então caracterizá-las.

A Tabela 1 apresenta a frequência da participação dos enfermeiros na construção dos discursos, representados pelas IC referentes à percepção da utilização de ferramentas gerenciais pelos participantes.

Tabela 1. Frequência da participação dos enfermeiros na construção dos discursos por ideia central sobre o tema conceito de ferramentas gerenciais, município do sul do Brasil, Brasil, 2013.

\begin{tabular}{l|r|r}
\hline Ideias centrais & $\mathbf{n}$ & $\%$ \\
\hline São meios de organização dos serviços de saúde & 11 & 29 \\
\hline É um direcionamento para o trabalho & 7 & 17 \\
\hline É o gerenciamento de pessoas & 6 & 15 \\
\hline São instrumentos padronizados de gestão & 5 & 14 \\
\hline Instrumentos para o gerenciamento da assistência & 4 & 10 \\
\hline Recursos para a avaliação do serviço & 3 & 9 \\
\hline Conhecimentos adquiridos durante a graduação & 2 & 3 \\
\hline Não tem definição sobre ferramenta gerencial & 2 & 3 \\
\hline Total & $\mathbf{4 0}$ & $\mathbf{1 0 0}$ \\
\hline
\end{tabular}

Nesta questão, a IC que obteve a maior participação de enfermeiros na construção do discurso foi a que definiu ferramenta gerencial como um meio de organização do serviço, seguidas pelas IC que a conceituaram como um direcionamento para o trabalho, como o gerenciamento de pessoas e que são instrumentos padronizados de gestão.

Os resultados evidenciaram que a maioria das respostas sobre a definição de ferramentas gerenciais foi relacionada ao fato de que elas são um meio para a organização dos serviços. Apesar do pouco número de respostas, alguns enfermeiros relacionaram as ferramentas gerenciais aos conhecimentos adquiridos na graduação e alguns não souberam definir o que seria uma ferramenta gerencial. Na sequência, apresenta-se a frequência da participação dos enfermeiros na construção dos discursos por ideia central sobre a utilização de ferramentas gerenciais na prática (Tabela 2). 
Tabela 2. Frequência da participação dos enfermeiros na construção dos discursos por ideia central sobre o tema utilização de ferramentas gerenciais na prática, município do sul do Brasil, Brasil 2013.

\begin{tabular}{l|r|r}
\hline Ideias centrais & $\mathbf{n}$ & \% \\
\hline Utilizo ferramentas gerenciais diariamente & 19 & 48 \\
\hline Utilizo poucas ferramentas gerenciais & 10 & 25 \\
\hline Utilizo ferramentas gerenciais empiricamente & 6 & 16 \\
\hline Não utilizo ferramentas gerenciais & 5 & 11 \\
\hline Total & $\mathbf{4 0}$ & $\mathbf{1 0 0}$ \\
\hline
\end{tabular}

Sobre a utilização ou não de ferramentas gerenciais, a maioria dos enfermeiros afirmou que as utiliza diariamente. Entretanto, 10 enfermeiros relataram utilizar poucas ferramentas, seis utilizam ferramentas gerenciais de forma empírica e outros cinco referem não utilizar.

A seguir, a Tabela 3 apresenta a frequência da participação dos enfermeiros nos discursos, representados pelas IC, referentes à questão sobre as ferramentas gerenciais utilizadas na prática profissional do enfermeiro na atenção básica.

Tabela 3. Frequência da participação dos enfermeiros na construção dos discursos por ideia central sobre as ferramentas gerenciais utilizadas na prática, município do sul do Brasil, Brasil, 2013.

\begin{tabular}{l|r|r}
\hline Ideias centrais & $\mathbf{n}$ & $\%$ \\
\hline Ferramentas para direcionamento do serviço & 13 & 32 \\
\hline Ferramentas de controle e avaliação de serviço & 7 & 17 \\
\hline Ferramentas para organização do serviço & 6 & 15 \\
\hline $\begin{array}{l}\text { Ferramentas gerenciais para o planejamento da } \\
\text { assistência }\end{array}$ & 5 & 13 \\
\hline Ferramentas relacionadas a competências gerenciais & 4 & 10 \\
\hline Desconheço as ferramentas gerenciais & 3 & 9 \\
\hline Faz uso empírico das ferramentas & 1 & 2 \\
\hline
\end{tabular}




\begin{tabular}{l|r|r}
\hline Ferramentas para gestão de custos & 1 & 2 \\
\hline Total & 40 & $\mathbf{1 0 0}$ \\
\hline
\end{tabular}

No que diz respeito ao tipo de ferramentas utilizadas por esses enfermeiros, a maioria (32\%) referiu o uso de ferramentas para direcionamento do serviço, o restante declarou o uso de ferramentas para o controle e avaliação do serviço, e de ferramentas para a organização do serviço. Poucos relatam utilizar ferramentas relacionadas a competências gerenciais e somente um enfermeiro refere o uso empírico e ferramentas para o gerenciamento de custos.

No quadro a seguir, são apresentados os temas norteadores, as questões e as IC com maior participação de enfermeiros nos discursos, num total de nove.

Quadro 2. Apresentação dos temas norteadores, questões e as IC da pesquisa sobre utilização de ferramentas gerenciais por enfermeiros da atenção básica, município do sul do Brasil, Brasil, 2013.

\begin{tabular}{|c|c|c|}
\hline Temas norteadores & Questões & Ideias centrais \\
\hline \multirow[t]{3}{*}{$\begin{array}{l}\text { Concepção de } \\
\text { ferramentas } \\
\text { gerenciais }\end{array}$} & \multirow[t]{3}{*}{$\begin{array}{l}\text { 1. O que é uma } \\
\text { ferramenta gerencial? }\end{array}$} & $\begin{array}{l}\text { IC 1: Ferramenta gerencial são } \\
\text { meios de organização dos } \\
\text { serviços de saúde }\end{array}$ \\
\hline & & $\begin{array}{l}\text { IC 2: Ferramenta gerencial é um } \\
\text { direcionamento para o trabalho }\end{array}$ \\
\hline & & $\begin{array}{l}\text { IC 3: Ferramenta gerencial é o } \\
\text { gerenciamento de pessoas }\end{array}$ \\
\hline \multirow[t]{6}{*}{$\begin{array}{l}\text { 2. Utilização das } \\
\text { ferramentas } \\
\text { gerenciais na prática }\end{array}$} & \multirow{3}{*}{$\begin{array}{l}\text { 2. Você utiliza } \\
\text { ferramentas gerenciais } \\
\text { no seu cotidiano de } \\
\text { trabalho? }\end{array}$} & $\begin{array}{l}\text { IC 4: Utilizo ferramentas } \\
\text { gerenciais diariamente }\end{array}$ \\
\hline & & $\begin{array}{l}\text { IC 5: utilizo poucas ferramentas } \\
\text { gerenciais }\end{array}$ \\
\hline & & $\begin{array}{l}\text { IC 6: Utilizo ferramentas } \\
\text { gerenciais empiricamente }\end{array}$ \\
\hline & \multirow{3}{*}{$\begin{array}{l}\text { 3. Quais são as } \\
\text { ferramentas gerenciais } \\
\text { que você utiliza na sua } \\
\text { prática profissional? }\end{array}$} & $\begin{array}{l}\text { IC 7: Ferramentas para } \\
\text { direcionamento do serviço }\end{array}$ \\
\hline & & $\begin{array}{l}\text { IC 8: Ferramentas de controle e } \\
\text { avaliação do serviço }\end{array}$ \\
\hline & & $\begin{array}{l}\text { IC 9: Ferramentas para } \\
\text { organização do serviço }\end{array}$ \\
\hline
\end{tabular}


No primeiro tema, "concepção de ferramentas gerenciais pelos enfermeiros entrevistados", serão apresentados os três discursos mais consistentes no que se refere à constituição dos mesmos, conforme prevê a técnica do DSC e suas IC. Assim, apresenta-se o DSC 1 referente à IC 1: "Ferramentas gerenciais são meios de organização dos serviços de saúde".

DSC 1 - São os artifícios e instrumentos que eu utilizo para gerenciar a unidade de saúde ou qualquer setor que trabalhe. Para organizar melhor o trabalho, facilitando o cotidiano, todos os planejamentos para organizar os programas, gerenciar a demanda, os funcionários, escala de trabalho. As ferramentas administrativas me ajudam a ver unidade de saúde como um todo. É um instrumento que auxilia a sistematizar o trabalho, os critérios para tomar decisões, apesar de ter que compartilhar com a gerente na unidade, qualquer decisão que eu queira tomar na unidade. São estruturas que eu utilizo como recursos materiais, humanos e tecnológicos.

O enfoque nesse discurso (DSC1) foi a coordenação e organização do serviço enquanto ferramentas gerenciais. O enfermeiro referiu também as duas funções administrativas, coordenação e organização, para a sistematização do trabalho gerencial que este executa. $O$ trabalho gerencial está focado na organização da unidade, da equipe e na tomada de decisão.

Na sequência da apresentação dos resultados, segue o segundo Discurso do Sujeito Coletivo (DSC2) que se refere à IC2: "Ferramenta gerencial é um direcionamento para o trabalho.

DSC2 - É uma forma para fazer, direcionar um trabalho, planejar as ações, o cuidado, a visita, o trabalho em equipe, para se aplicar a gerência, todos os instrumentos do enfermeiro, na parte gerencial, não só assistencial. É um norte, um direcionamento, é saber fazer gestão das unidades. São métodos que nos apoiam e auxilia a desenvolver o trabalho, uma escala, uma rotina, um manual. Sem essa linha, esse direcionamento eu não consigo executar aquilo que pretendo, fico muito travado.

No DSC2, emergiu a ferramenta gerencial como um método de trabalho norteador das ações administrativas e gerenciais. Dos depoimentos surgiu o planejamento como direcionador dessas ações do enfermeiro. Esse discurso torna evidente a necessidade de ferramentas gerenciais na gestão do cuidado, indicadas para organizar a assistência prestada na unidade em que ele é responsável. Outro fator ressaltado no discurso é o trabalho em equipe. 
A terceira IC apresentada é a IC 3: "Ferramenta gerencial é o gerenciamento de pessoas e o seu discurso correspondente".

\begin{abstract}
DSC3 - Ferramenta gerencial é um instrumento para gerenciar os profissionais da unidade e posto. É administrar e conduzir uma equipe sem impor as coisas, o papel de cada funcionário, de cada regulador do serviço, na hora de explicar ou chamar a atenção, receber também orientação das outras pessoas da equipe. Um método de trabalho para planejar a supervisão de outros profissionais. A capacitação da equipe, reconhecimento do funcionário pela pontualidade, uma gratificação para o funcionário, ideal é que a empresa já tenha uma ferramenta já padronizada para isso.
\end{abstract}

O sujeito coletivo do DSC3 referiu à percepção da ferramenta gerencial como instrumento que auxilia na gestão de pessoas, principalmente na condução do trabalho da equipe, supervisão e orientação. O DSC3 associa a ferramenta a um método de trabalho, capacitação e estimulação da motivação por meio de recompensa do funcionário. Deste discurso emerge também a necessidade de padronização de ferramentas gerenciais pelas instituições.

Ainda na questão sobre o uso de ferramentas no cotidiano de trabalho, no segundo tema/segunda questão, na qual se perguntou ao enfermeiro sobre a utilização ou não de ferramentas gerenciais, apresentam-se as três IC que emergiram do material verbal com número mais significativo de participação de enfermeiros na construção dos discursos. Conforme se observa a seguir, a primeira IC deste tema é a IC 4: "Utilizo ferramentas gerenciais diariamente" e o discurso.

DSC 4 - Eu uso diariamente, preciso utilizar as ferramentas para o bom desenvolvimento da assistência. Aqui, nós somos de tudo um pouco, gerente, supervisor, assistencial. Tenho que planejar meu trabalho e através disso eu vou usar o instrumento como o perfil epidemiológico, da minha população. A gente usa os relatórios dos programas, a avaliação, que é uma das coisas gerenciais. Protocolos de pré-natal, hiperdia e de outras rotinas do serviço, eu preciso monitorar. São instrumentos para planejar o meu trabalho e direcionar as minhas ações.

Neste discurso, o sujeito considerou importante o uso de ferramentas para o desenvolvimento do cuidado. Referiu uso e transposição das várias dimensões do processo de trabalho do enfermeiro para esse gerenciamento e houve certa confusão com o que seria ferramentas (supervisão) e dimensões (ou subprocessos) do trabalho do enfermeiro, função gerencial e assistencial. 
Ainda na segunda questão, apresenta-se o discurso do sujeito coletivo referente à IC 5: "Utilizo poucas ferramentas gerenciais", no tema utilização de ferramentas no cotidiano:

DSC 5 - Aqui na saúde pública tem muito pouco. No hospital eu trabalhava com ferramentas de gerenciamento, aqui é pouco, o que eu utilizo é o prontuário com o formulário. Na unidade a gente utiliza escala de trabalho, organização de processo de trabalho, manual de normas e rotinas, instrumentação de trabalho. Em relação o atendimento aos pacientes, prioridade e encaixe para o médico.

O sujeito coletivo nesse discurso descreveu que as ferramentas gerenciais não são sistematizadas na Atenção Básica, e aponta algumas ferramentas que utiliza: escala de trabalho, organização de processo de trabalho, manual de normas e rotinas e informações. Além destas, citou o que ele considerou ferramentas para o atendimento ao usuário: triagem, encaminhamento e encaixe deste para o atendimento médico. O sujeito coletivo enfocou que, como não há ferramentas sistematizadas de gestão, também usa ferramentas próprias para a gestão de pessoas.

A ideia central 6 (IC 6): "Utilizo ferramentas gerenciais empiricamente", referese ainda à segunda questão, cujo discurso é apresentado a seguir.

DSC 6 - Eu uso ferramentas empiricamente. Acho que não está bem registrado, não está bem definido. A gente acaba trabalhando e sabe que está usando, mas tem coisas que poderiam estar no papel. A gente não para e pensa nisso, mas a gente tem que utilizar, se não como é que você vai planejar. Às vezes eu não a utilizo da forma que é, mas a gente a utiliza dia a dia, toda dia, toda hora, porque tudo que eu estou falando, uma orientação, tudo é uma ferramenta de gerenciar.

Neste discurso (DSC 6) o sujeito coletivo sabe que utiliza as ferramentas, mas não consegue pontuar quais. Para esse sujeito o uso consciente de ferramentas está relacionado com a formalização dessas no serviço, referindo que elas estão apenas "no papel".

Sobre a terceira questão, quais ferramentas são utilizadas, ainda dentro do tema utilização de ferramentas gerenciais, serão apresentados os discursos mais relevantes, conforme o número de enfermeiros em sua composição. Apresenta-se a primeira ideia central da questão, a IC 7: "Ferramentas para organização do serviço". 
DSC7 - As reuniões, a conversa individual um com o outro para gente poder organizar o trabalho. O planejamento, o gerenciamento do serviço no sentido de programar rotina. As agendas, principalmente a agenda da parte da enfermeira, porque nós temos os dias específicos para fazer cada atividade.

O DSC7 destacou ferramentas como reunião, planejamento, rotinas, agendas para organizar o serviço, as quais auxiliam o enfermeiro a planejar e a organizar a sua prática de forma mais reflexiva, fugindo do "apaga incêndio", em que as ações de enfermagem são realizadas conforme acontecem, sem tempo para reflexão e planejamento. Esse discurso contradiz o DSC 6 em que os participantes referiram utilizar ferramentas gerenciais de forma empírica, sem conhecimento e reflexão sobre essas.

O enfermeiro relatou que também utiliza a ferramenta comunicação, quando relata a conversa individual com cada um da sua equipe, evidenciando um planejamento participativo e um rompimento, mesmo que incipiente do modelo de gerência tradicional. Destaca-se a importância de observar este tipo de ferramenta no contexto da Atenção Básica, em que o trabalho interdisciplinar e em equipe são primordiais para o atendimento das necessidades de saúde da população.

No seguimento da apresentação dos resultados, a segunda ideia central da última questão, quais as ferramentas utilizadas pelos enfermeiros da atenção básica, é a IC 8: "Ferramentas de controle e avaliação do serviço e o seu discurso".

DSC 8 - Os relatórios com datas, tem relatório semanal e mensal do prénatal, hiperdia, dos agentes comunitários de saúde, controle diário deles, o relatório mensal da enfermagem. Eu utilizo fichas, formulários, impressos, planilhas mesmo já pré-elaboradas pela Secretaria e outras que a gente mesmo montou na unidade e os livros de ocorrência e anotação.

O foco neste discurso é o cumprimento de metas pré-estabelecidas pelas autoridades da atenção à saúde do município prevalecendo à questão da produção e controle, herança dos modelos Taylorista/fordista, ainda muito presente na gestão em saúde nos dias atuais. Enfatiza-se a avaliação por meio da produção num determinado período pelos órgãos superiores, o que corrobora o enfoque da Administração Cientifica.

O último discurso é ainda do segundo tema e da terceira questão, e a sua ideia central é a IC 9: Ferramentas para direcionamento do serviço. 
DSC 9: Protocolos, memorando, normativas, manuais de normas e rotinas de enfermagem, documentação para gerenciar um serviço. Cronograma, fluxograma das ações, elaboração das escalas, escalas de grupos, de atividades educativas, escala de serviço, as escalas de vacina, de quem verifica a temperatura da geladeira.

O sujeito coletivo DSC 9 relatou a realização de escalas de atividades e escala de férias como dimensionamento, evidenciando uma confusão conceitual sobre as atividades. Ainda mencionou como ferramentas gerenciais: protocolos, memorandos, normas, rotinas e cronogramas, enfatizando uma prática pautada na lógica da valorização dos processos de trabalho, marca da administração clássica.

\section{DISCUSSÃO}

Os resultados evidenciam que o foco dos enfermeiros que atuavam na atenção básica estava na organização do serviço para o atendimento ao usuário. Este fato foi observado em pesquisas realizadas com enfermeiros da estratégia saúde da família, em que esse profissional assume o papel de gerente no processo de trabalho das Unidades de Saúde, que coordena, administra e gerencia o trabalho das unidades e dos membros da equipe ${ }^{5,16}$.

Contudo, cabe salientar que a efetividade do processo de trabalho no gerenciamento de enfermagem também está vinculada não só à organização da unidade, mas também à educação de toda a equipe de enfermagem a fim de qualificar o cuidado e os serviços prestados ao usuário. Assim, cabe aos gestores oferecer viabilidade ao processo de gerenciamento por meio da organização da estrutura, dos objetivos, da missão e da cultura da instituição ${ }^{17,18}$.

Os enfermeiros afirmaram que as ferramentas gerenciais são artifícios utilizados também para o direcionamento do trabalho. Para isso, o instrumento/ferramenta gerencial é um ponto chave, estratégico, e pode ser utilizado como um modelo para direcionar os indivíduos na execução, adequação e avaliação do cuidado, a fim de favorecer e elevar o nível de qualidade e resolutividade dos serviço ${ }^{11}$.

Sabendo-se que o cuidado é o que permeia o trabalho do enfermeiro, cabe ressaltar que o uso do processo de enfermagem para a sistematização do cuidado é uma ferramenta que organiza e consequentemente direciona, qualifica e quantifica a assistência, controlando os custos e facilitando a auditoria, o que permite $o$ alcance das metas relacionadas à qualidade do cuidado prestado ${ }^{18,19}$.

Para uma efetiva organização e um correto direcionamento das ações, faz-se necessário que o enfermeiro conduza a equipe para o alcance de objetivos comuns, reconhecidos por cada membro do grupo. Por esse motivo, um ponto 
importante no trabalho gerencial dos enfermeiros é o gerenciamento da equipe de enfermagem e de saúde, segundo o que diz o relatório da Organização Mundial de Saúde $(\mathrm{OMS})^{20}$.

Uma pesquisa sobre a gestão de pessoas em um hospital do Sistema Único de Saúde aborda que os desafios nesta gestão influenciam nas instituições que compõe a rede, onde cada uma responde a esses desafios de maneira individual de acordo com sua organização e estruturação interna ${ }^{21}$. Para isso, existem três estratégias para os desafios encontrados no gerenciamento de pessoas: melhorar o recrutamento, auxiliar a força de trabalho a melhorar seu desempenho e diminuir a rotatividade dos trabalhadores ${ }^{20}$.

Portanto para todo o processo de trabalho do enfermeiro, seja ele na organização ou no direcionamento dos serviços, ou até mesmo na gestão de pessoas, faz-se necessário o uso de ferramentas gerenciais como recurso de auxílio para se alcançar os objetivos traçados. Alguns autores destacam a utilização destas ferramentas principalmente no processo gerencial do enfermeiro, no qual uma das atividades fundamentais desse profissional é o planejamento das ações, para que elas sejam feitas de maneira ordenada para atingir os resultados esperados ${ }^{17,22}$.

O planejamento, entendido como instrumento do processo de trabalho gerencial, pode ser definido como um modo de fazer escolhas e de elaborar planos para favorecer um processo de mudança. Porém, muitas escolhas, são aleatórias, sem reflexão ou consciência do processo ou das etapas percorridas para se fazer essas escolhas e tomar decisões ${ }^{23}$.

Em contrapartida, quando se fala em ferramentas gerenciais, muitos enfermeiros ainda pensam em questões burocráticas, principalmente quando se trata de atenção básica que possui inúmeros relatórios e formulários, e para os quais o enfermeiro se tornou o profissional responsável pelo preenchimento. Com a organização da enfermagem no século XX, houve uma transformação onde a adoção de rotinas e protocolos de organização distanciaram o profissional do paciente, fazendo com que as rotinas e métodos se tornassem mais importantes que a finalidade do trabalho, ou até mais importantes que o agente $^{16,22}$.

Porém em alguns casos, estes utilizam essas ferramentas de maneira empírica, dados encontrados também em uma pesquisa, cujo resultado identificou que este empirismo, sem reflexão sobre o seu uso ${ }^{7}$.

As ferramentas gerenciais são indispensáveis para o processo de cuidado, tendo como propósito organizar, padronizar, direcionar e comunicar a estrutura e o funcionamento dos serviços. No entanto, para que este funcionamento aconteça, é importante o uso de ferramentas capazes de mensurar o quantitativo de pessoal necessário para um cuidado efetivo e de organizar a distribuição destes profissionais da melhor maneira possível. Apesar de ser visto comumente em ambientes hospitalares, o dimensionamento de pessoal também necessita ser valorizado como ferramenta gerencial na atenção básica ${ }^{24}$. 
Vale destacar que o dimensionamento de enfermagem é a etapa inicial do processo de provimento de pessoal, que tem como finalidade a previsão da quantidade de funcionários por categoria, requerido para suprir as necessidades do cuidado de enfermagem, direto ou indiretamente prestado à clientela ${ }^{25}$. Apesar de antigo, esse conceito ainda é o mais utilizado na literatura brasileira ${ }^{26 .}$

Outro ponto nos discursos é o uso das ferramentas que organizam os serviços como reuniões e planejamento, das mais simples e empíricas às mais sofisticadas, como as que facilitam a comunicação. A comunicação é fundamental para o desenvolvimento do trabalho dos enfermeiros junto à equipe e a pacientes atendidos nas instituições e para a transmissão de uma informação universal, além de exercer influência direta sobre os indivíduos ${ }^{11,27}$.

\section{CONCLUSÃO}

Os resultados apontam para uma fragilidade no tocante à prática gerencial dos enfermeiros na atenção básica, e que esses se encontram sobrecarregados de funções administrativas, além das assistenciais. A ferramenta gerencial pode ser um recurso valioso para auxiliá-lo a desempenhar suas funções gerenciais de forma efetiva e com qualidade, que podem organizar e agilizar as ações do enfermeiro, nas várias dimensões de seu trabalho: gerência, assistência, educação e pesquisa.

Percebe-se que as dificuldades apresentadas pelos participantes em reconhecer e utilizar ferramentas que auxiliem no processo gerencial podem ser devidas ao baixo conhecimento sobre esse tema e ainda, a questão da sobrecarga de atividades que possam ser delegadas, caso, utiliza-se uma técnica de planejamento das atividades de cuidado e das questões burocráticas que conforme os discursos absorvem muito seu tempo.

Uma conclusão dos resultados da pesquisa é que, apesar dos enfermeiros entenderem que necessitam desenvolver as competências gerenciais e conhecer ferramentas de gestão para auxiliá-lo no processo gerencial, torna-se necessário que este profissional se desvincule das ações embasadas apenas no conhecimento empírico e torne a sua prática crítica e reflexiva.

A presente pesquisa não teve a pretensão de esgotar o assunto e tratou de um cenário específico da prática do enfermeiro. Ademais, cabem futuras pesquisas com vistas a fornecer informações sobre ferramentas gerenciais específicas que possam ser utilizadas nos diversos âmbitos de sua prática profissional.

Por esse motivo, atualmente, espera-se que os profissionais de saúde desenvolvam conhecimentos para promover o cuidado de qualidade utilizando, para isso, ferramentas gerenciais e competências que o apoiem nas tomadas de decisão, sendo a capacidade de comunicação, a organização, o planejamento e a boa gestão de pessoas fundamentais para esse processo ${ }^{28}$. 


\section{REFERÊNCIAS}

1. Backes DS, Backes MS, Erdmann AL, Büscher A. O papel profissional do enfermeiro no Sistema Único de Saúde: da saúde comunitária à estratégia de saúde da família. Rev Ciência \& Saúde Coletiva. 2012; 17(1):223-230.

2. Lorenzetti J, Oro J, Matos E, Gelbcke FL. Organização do trabalho da enfermagem hospitalar: abordagens na literatura. Texto Contexto Enferm [Internet]. 2014 [cited 2016 Dec 01]; 23 (4): 1104-12. Available from: <http://www.redalyc.org/articulo.oa?id=71433508034> ISSN 0104-0707.

3. Osorio Castaño JH. Patrón de conocimiento socio-político en enfermería: reflexiones conceptuales. Rev Cuid [Internet]. 2016 [cited 2017 Feb 01]; 7 (2): 1352-7. Available from:

<https://www.revistacuidarte.org/index.php/cuidarte/article/view/319> . ISSN 2216-0973.

4. Christovam BP, Porto IS, Oliveira DC. Gerência do cuidado de enfermagem em cenários hospitalares: a construção de um conceito. Ver Esc Enferm USP [Online]. 2012 [cited 2016 Nov 15]; 48 (8): 734-41. Available from: http://www.revistas.usp.br/reeusp/article/view/41004/44544.

5. Freitas GM, Santos NSS. Atuação do enfermeiro na atenção básica de saúde: revisão integrativa de literatura. $R$ Enferm Cent $O$ Min [Internet]. 2014 [cited 2017 Mar 17]; 4 (2):1194-1203. Available from: http://www.seer.ufsj.edu.br/index.php/recom/article/view/443/754doi:10.19 175/recom.v0i0.443

6. Fernandes MC, Silva FMP, Costa SP, Andrade ME. Facilities managers and difficulties of nurses in the implementation of environmental management of care hospital. Revista de Pesquisa: Cuidado é Fundamental Online. 2016; 8(4), 5039-5044.

7. Almeida ML, Segui MLH, Maftum MA, Labronici LM. Instrumentos gerenciais utilizados na tomada de decisão do enfermeiro no contexto hospitalar. Texto \& Contexto Enfermagem (UFSC. Impresso). 2011 [Cited 2013 Jan 20]; 20 (Esp): 131-137.

8. Chaves LDP, Tanaka, OY. O enfermeiro e a avaliação na gestão de Sistemas de Saúde. Rev. Esc. Enferm. USP [Online]. 2012 [Cited 2016 Dec 20]; 46 (5): $1274-1278$. Available from: http://www.revistas.usp.br/reeusp/article/view/48154 ISSN 0080-6234.

9. Castro JL. Nova agenda para a administração de recursos humanos de saúde. Disponível 
em:http://www.opas.org.br/rh/publicacoes/textos_apoio/pub04U3T3.pdf. Acesso em 22/01/2013 às 22:44 horas.

10. Vale EG, Oliveira SC. Ferramentas de gestão em enfermagem. In: Programa de Atualização em Enfermagem (PROENF):

Gestão/Organizadora-geral: Eucléia Gomes Vale; Organizadoras: Simone Aparecida Peruzzo, Vanda Elisa Andres Felli. - Porto Alegre: Artmed/ Panamericana, 2011.

11. Carvalho BG, Peduzzi M, Nunes EFPA, Leite FS, Silva JAM. Gerência de unidade básica de saúde em municípios de diferentes portes: perfil e instrumentos gerenciais utilizados. Rev. Esc. Enferm. USP [Online]. 2014 [Cited 2017 Mar 05]; 48 (5): 907-914. Available from: http://www.journals.usp.br/reeusp/article/view/103090.

12. Ruthes RM, Feldman LB, Cunha ICKO. Foco no cliente: ferramenta essencial na gestão por competência em enfermagem. Rev. bras. enferm. [online]. 2010 [Cited 2013 Jan 20]; 63 (2): 317-321. Available from: ISSN 0034-7167. http://dx.doi.org/10.1590/S0034-71672010000200023.

13. Houaiss A, Villar MS. Dicionário Houaiss da língua portuguesa/ Antônio Houaiss e Mauro de Sales Villar, elaborado pelo Instituto Antônio Houaiss de Lexicografia e Banco de Dados da Língua Portuguesa S/C Ltda - Rio de Janeiro: Objetiva 2009.

14. Lefevrè $F$, Lefevrè AMC. Pesquisa de Representação Social. Um enfoque qualiquantitativo. A metodologia do Discurso do Sujeito Coletivo. Brasília: Liber livro; 2010.

15. Lefevrè F, Lefevrè AMC; Simioni AAC; Ferraz MTM. Discurso do Sujeito Coletivo: Qualiquantisoft passo a passo. São Paulo (SP): Instituto de Pesquisa do Discurso do Sujeito Coletivo (IpDsc), 2009.

16. Galavote HS, Zandonade E, Garcia ACP, Freitas PSS, Seidl H, Contarato, $\mathrm{PC}$, et al. $\mathrm{O}$ trabalho do enfermeiro na atenção primária à saúde. Esc. Anna Nery [online]. 2016 [Cited 2017 Mar 10]; 20 (1): 90-98. Available from: <http://www.scielo.br/scielo.php?script=sci_arttext\&pid=S1414$81452016000100090 \&$ lng $=$ pt\&nrm=iso $>$. ISSN $1414-$ 8145. http://dx.doi.org/10.5935/1414-8145.20160013.

17. Giordani JN, Bisogno SBC, Silva LAA. Percepção dos enfermeiros frente às atividades gerenciais na assistência ao usuário. Acta Paul. Enferm. [online]. 2012 [Cited 2016 Nov 17]; 25 (4): 511-516. Available from: http://www2.unifesp.br/acta/pdf/v25/n4/v25n4a5.pdf. ISSN 01032100 .

18. Diniz IA, Cavalcante RB, Otoni A, MATA LRF. Percepção dos enfermeiros gestores da atenção primária sobre o processo de enfermagem. Rev. Bras. Enferm. [online]. 2015 [Cited 2016 Dec 10]; 68 (2): 206-213. Available from: http://oaji.net/articles/2015/6721438870084.pdf. ISSN 1984-0446. 
19. Santos JLG, Pestana AL, Guerrero P, Meirelles BSH, Erdmann AL. Práticas de enfermeiros na gerência do cuidado em enfermagem e saúde: revisão integrativa. Rev. Bras. Enferm. [online]. 2013 [Cited 2016 Mai 06]; 66 (2): 257-263. Available from: http://search.proquest.com/openview/216a1e83dfa94f02d5975e61460ff90f 11 ?pq-origsite $=$ gscholar \&cbl=2032618. ISSN 0034-7167.

20. Organização Mundial da Saúde (OMS). Trabalhando juntos pela saúde. Relatório Mundial de Saúde 2006. Brasília: Ministério da Saúde, 2007.

21. Morici MC, Barbosa ACQ. A gestão de Recursos Humanos em hospitais do Sistema Único de Saúde (SUS) e sua relação ao modelo de assistência: um estudo em hospitais de Belo Horizonte, Minas Gerais. Revista de Administração Pública [Impresso]. 2013 [Cited 2014 Dec 10]; 47 (1): 205-225.

22. Felli VE, Peduzzi M. $O$ trabalho gerencial do enfermeiro. In: Kurcgant $P$, Trochin DMR, Fugulin FMT, Peres HHC, Massarollo MCKB, Fernandes MFP et al. Gerenciamento em enfermagem. Rio de Janeiro (RJ): Guanabara Koogan; 2010.

23. Ciampone MHT, Melleiro MM. O planejamento e o processo decisório como instrumentos do processo de trabalho gerencial. In: Kurcgant $P$, organizador. Gerenciamento em enfermagem. $2^{\underline{a}}$ Ed. Rio de Janeiro (RJ): Guanabara Koogan; 2010.

24. Bonfim D, Gaidzinski RR, Santos FM, Souza Gonçales C, Fugulin, FMT. Identificação das intervenções de enfermagem na Atenção Primária à Saúde: parâmetro para o dimensionamento de trabalhadores. Rev Esc Enferm USP [Online]. 2012 [Cited 2016 Jun 05]; 46 (6), 1462-1470. Available from: http://www.revistas.usp.br/reeusp/article/view/5283.

25. Kurcgant $P$, Cunha $K$, Gaidzinski RR. Subsídios para a estimativa de pessoal de Enfermagem. Enfoque, v. 17, n. 3, p. 79-81, 1989.

26. Fugulin FMT, Gaidzinski RR, Castilho V. Dimensionamento de pessoal de enfermagem em instituições de saúde. In: Kurcgant $P$, (org). Gerenciamento em enfermagem. $2^{\mathrm{a}} \mathrm{Ed}$. Rio de Janeiro (RJ): Guanabara Koogan; 2010.

27. Broca PV, Ferreira MA. Action plan for the comunication process in a nursing team. Acta Scientiarum [Internet]. 2016 [Cited 2017 Jan 10]; 38 (1): 23-31. Available from: http://periodicos.uem.br/ojs/index.php/ActaSciHealthSci/article/view/29758

Recebido: 11 novembro 2017. Publicado: 04 dezembro 2017 
Correspondência: Samuel Andrade de Oliveira. E-mail: enf.samuelandrade@gmail.com

Conflito de Interesses: os autores declararam não haver conflito de interesses.

(C) This is an Open Access article distributed under the terms of the Creative Commons Attribution License, which permits unrestricted use, distribution, and reproduction in any medium, provided the original work is properly cited 